\section{Blood Donor Leukocyte Reduction Filters as a Source of Human B Lymphocytes}

\section{BioTechniques 31:464-466 (September 2001)}

Human lymphocytes are widely used in biomedical research. Our laboratory collects human $\mathrm{B}$ cells to study $\mathrm{B}$ cell immunoglobulin gene repertoire usage or to generate human B cell hybridomas. The absolute amount of $\mathrm{CD} 19^{+} \mathrm{B}$ cells in healthy adults is approximately $3 \times 105 / \mathrm{mL}$ blood (1) However, the average yield of CD19+ B cells generated by the separation of peripheral blood mononuclear cells (PBMC) from whole blood by density gradient centrifugation and subsequent immunomagnetic isolation is $4-6 \times$ $10^{4} / \mathrm{mL}$ blood. Many experiments require the use of $40 \times 10^{6} \mathrm{~B}$ cells that would require from 700 to $1000 \mathrm{~mL}$ peripheral blood from healthy adults. Frequent large blood donations from volunteers are difficult to obtain on a regular basis and are costly. Here, we describe a procedure using a simple, inexpensive, and readily available source for the generation of large quantities of human lymphocytes by back-flushing leukocyte reduction filters from American Red Cross blood donations.

Disposable leukocyte reduction filters are used daily by the Red Cross to prepare leukocyte-poor red blood cell transfusion units. The Red Cross in Nashville, TN, provides us with used RCM1 Leukocyte Reduction Filters (PALL, Birmingham, AL, USA) for a minor fee. Each filter is used for prestorage leukoreduction of red cells from peripheral blood donations of 500 $\mathrm{mL}$ average volume by overnight application using gravity flow. After use, the tubing to and from the filter is crimped and sealed, and the excess tubing is cut to remove the in-line filter. Crimped filters are stored for less than $8 \mathrm{~h}$ at $4^{\circ} \mathrm{C}$ before transport to our laboratory. We use a $30-\mathrm{mL}$ syringe filled three times with PBS containing $5 \mathrm{mM}$ EDTA to back-flush the filters (Figure 1), collecting approximately $80 \mathrm{~mL}$ blood cell suspension from each filter. We subsequently separate PBMC by overlaying
$15 \mathrm{~mL} 1.078$ density lymphocyte separation medium (Cellgro ${ }^{\circledR}$; Mediatech, Herndon, VA, USA) with 40 mL PBSEDTA cell suspension in each of two
50-mL conical tubes, followed by centrifugation at $450 \times g$ for $35 \mathrm{~min}$ at room temperature. Collection of the interface yields on average $1.5 \times 10^{8} \mathrm{PBMC}$

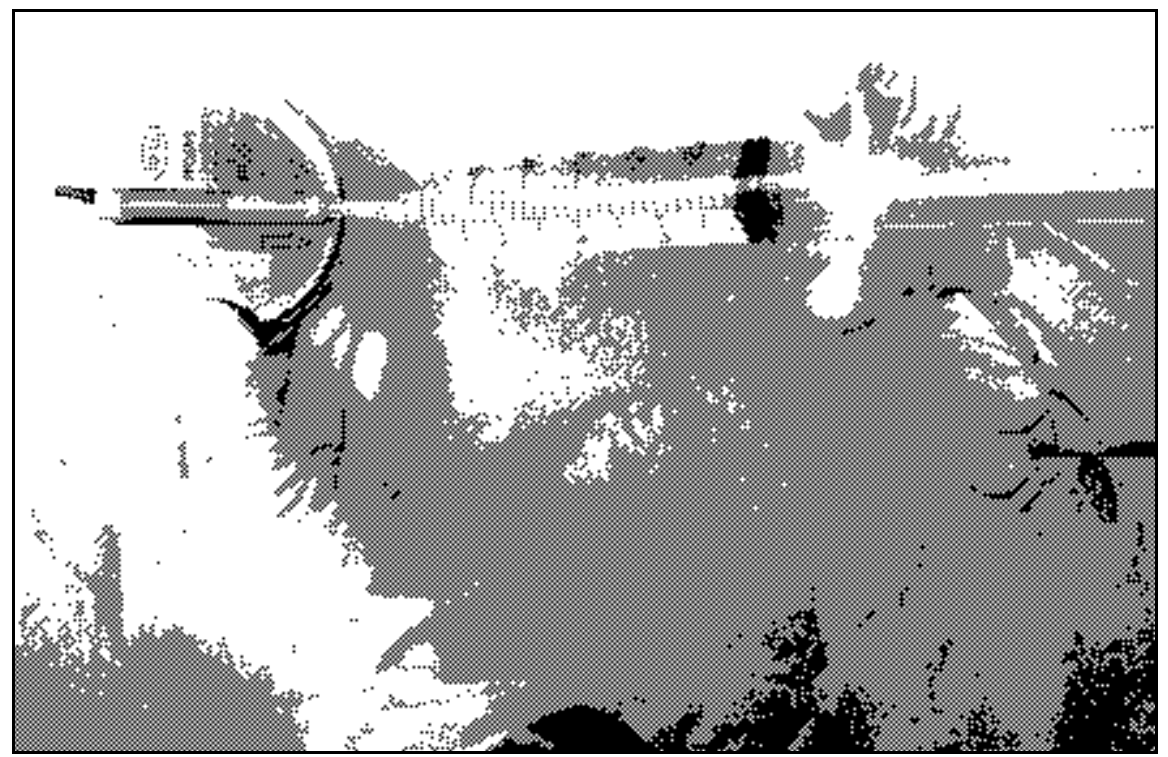

Figure 1. Back-flushing the leukocyte reduction filter, using a 30-mL syringe filled with PBS containing 5 mM EDTA.
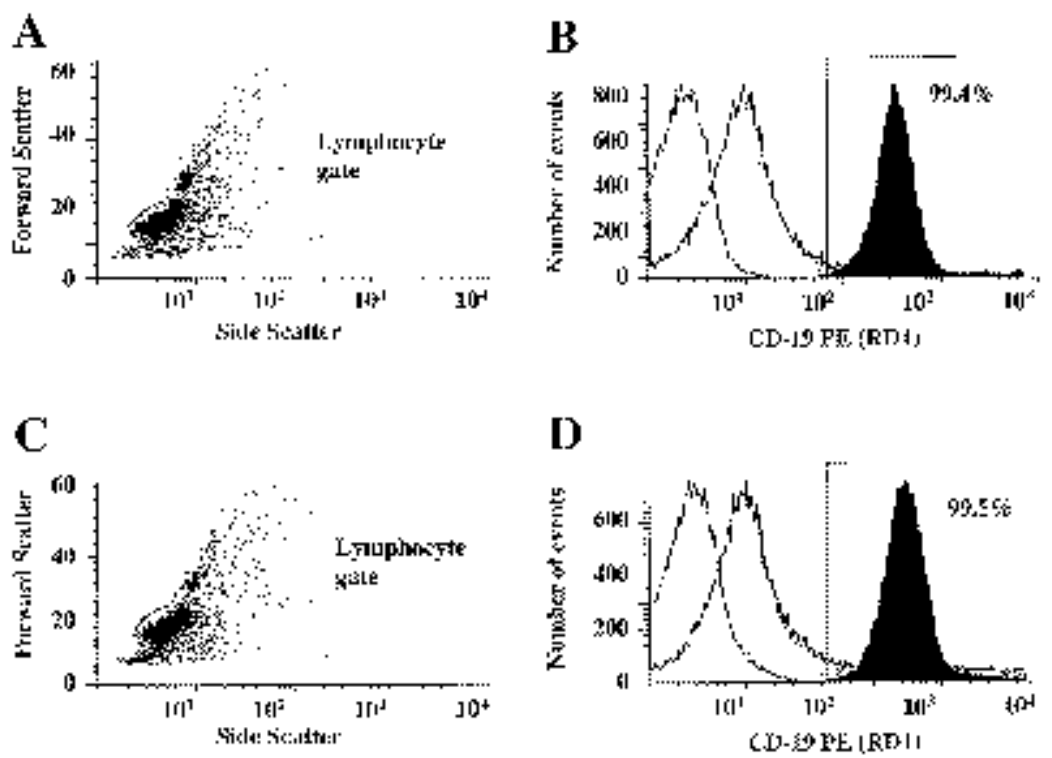

Figure 2. Flow cytometric analysis of magnetically selected putative $\mathrm{CD}^{+}{ }^{+} \mathrm{B}$ cells from a leukocyte reduction filter (A and B) or from fresh human blood (C and D). Lymphocytes from a leukocyte reduction filter (A) or from fresh blood (C) were gated by forward and side light scatter, then gated cells were analyzed for CD-19 PE (RD1) fluorescence. As shown by an overlay histogram (B and D), CD19+ B cells were identified by increased CD-19 PE (RD1) fluorescence (black), compared to unstained B cells (open) or B cells stained with an isotype-matched control reagent (gray). For each sample, 25000 events were collected. 99.4\% of gated lymphocytes from the leukocyte reduction filter (B) and $99.5 \%$ of gated lymphocytes from fresh human blood (D) were considered CD19+. 
from each filter (range 0.5 to $1.9 \times 10^{8}$ of nine filters tested). Separation of PBMC with paramagnetic beads conjugated with anti-CD19 monoclonal antibodies (Dynabeads ${ }^{\circledR}$; Dynal, Lake Success, NY, USA) results in an average yield of $9.5 \times 10^{6}$ putative $\mathrm{CD} 19^{+} \mathrm{B}$ cells from each filter (range 2 to $27 \times$ $10^{6}$ of 50 filters tested). Virtually all of the selected cells (>99\%) appeared to be viable by trypan blue dye exclusion. We performed flow cytometry analysis of the cells with a FACStarPlus ${ }^{\circledR}$ flow cytometer and Cellquest ${ }^{\circledR}$ software (Becton Dickinson, Franklin Lakes, NJ, USA). Analysis by forward and side scatter parameters (Figure 2A) suggested that the quality of filter-derived $B$ cells was similar to cells isolated by the same technique from fresh human blood (Figure 2C). Cells that were isolated from either a filter or fresh human blood using CD-19-specific Dynabeads were labeled with anti-CD-19 PE (Cyto-stat ${ }^{\circledR} /$ Coulter Clone ${ }^{\circledR}$ B4-RD1; Coulter, Miami, FL, USA). Flow cytometry analysis showed that greater than $99 \%$ of gated lymphocytes from a filter or from fresh human blood were CD19+, using parameters established by comparison with unstained controls or cells stained with an isotypematched control reagent (Cyto-stat/ Coulter Clone MsIgG1-RD1) (Figure 2 , B and D).

Universal precautions are always indicated when using blood derived from human volunteers. However, our method allows an added measure of safety because the Red Cross prescreens blood donors by interview for risk factors for common blood-borne pathogens. Furthermore, the Red Cross performs specific testing for hepatitis $\mathrm{B}$, hepatitis $\mathrm{C}$, and human immunodeficiency virus infection on blood from which cells can be derived. The Red Cross makes available to us the results of adventitious agent testing the day following collection. The donor's identity is protected because patient-specific identifiers are never released to the research laboratory.

In summary, using blood donor leukocyte reduction filters with a sim ple back-flush manipulation provides a safe, inexpensive, and readily available source for large numbers of human lymphocytes.

\section{REFERENCE}

1.Hannet, I., F. Erkeller-Yuksel, P. Lydyard, V. Deneys, and M. DeBruyère. 1992. Developmental and maturational changes in human blood lymphocyte subpopulations. Immunol. Today 13:215-218.

We thank Catherine E. Allen for excellent flow cytometry assistance and Dr. James $O$. Price for help in analyzing the flow cytometry data. This work was supported by NICHD, National Institutes of Health grant no. R01 HD36311 (to J.E.C.) and Deutsche Forschungsgemeinschaft Program Award WE 2405/1-1 (to J.-H.W.). Address correspondence to Dr. James E. Crowe, Jr., Vanderbilt University Medical Center, Department of Pediatrics, Division of Pediatric Infectious Diseases, D-7235 Medical Center North, 116121 st Avenue South, Nashville, TN 37232-2581, USA. e-mail: james.e.crowe@vanderbilt.edu

Received 26 December 2000; accepted 29 May 2001.

Jörn-Hendrik Weitkamp and James E. Crowe, Jr. Vanderbilt University Medical Center

Nashville, TN, USA

\section{Improved Efficiency and Reliability of RT-PCR Using Tag-Extended RT Primers and Temperature Gradient PCR}

BioTechniques 31:466-472 (September 2001)

In RT-PCR on prokaryotic RNA, an often-encountered problem is the contamination of RNA with chromosomal DNA, which generates false-positive reaction products. Over the past decade, a number of methods has been developed to improve the reliability of RT-PCR. In some of the methods, DNA is degraded by an RNase-free DNase. However, extensive DNase treatment at relatively high temperatures leads to the loss of mRNA, and thus a lower specificity and sensitivity of the analysis $(2,6)$. In other methods, DNase treatment can be omitted because of the generation of different denaturation temperatures of the dsRNA/DNA hybrid as compared to dsDNA (1) or the development of primers that display differential annealing behavior during $\mathrm{RT}$ and the subsequent PCR $(4,5,7,10)$. Shuldiner et al. (10) describe the development of primers that generate 5'tagged cDNA during RT that is subsequently used as a specific template during PCR. Because the sequence of this $5^{\prime}$ tag is not present in chromosomal DNA, no DNA-derived product is amplified during PCR. Koo et al. (7) describe the development of a primer containing mismatched bases. Consequently, this primer can only anneal at relatively low temperatures, similar to those applied during RT. Subsequent PCR involves higher annealing temperatures, which prevent the primer from annealing to the chromosomal DNA because of its mismatched base pairs. The convenient application of both methods is impaired by the development of long primers (47 bp) (10) and/or by the laborious optimization of the experimental conditions. Primers often need to be optimized and resynthesized several times, and annealing temperatures need to be optimized in many successive PCR runs. Another drawback of these methods is the increased amount of false-positive reaction products when increasing concentrations of contaminating chromosomal DNA are present in the RNA samples. Therefore, the reproducibility of RTPCR results is also limited when different batches of RNA isolates are used. Here, we describe the application of temperature gradient PCR to improve the efficiency and reliability of an RTPCR method using tag-extended primers. The methodology is a valuable extension to the previously described method. The use of temperature gradient PCR makes it possible to visualize the functionality of the primers in simultaneously performed PCR amplifications with increasing annealing temperatures and thus guarantees that 